\title{
Experience-Dependent Activation Patterns in Human Brain during Visual-Motor Associative Learning
}

\author{
James C. Eliassen, Timothy Souza, and Jerome N. Sanes \\ Department of Neuroscience, Brown Medical School, Providence, Rhode Island 02912
}

\begin{abstract}
Multiple brain regions, including parietal and frontal cortical areas, seem to participate in learning and rehearsing associations between spatially defined visual cues and appropriate motor responses. However, because most previous studies have related learning to changes in brain activation according to elapsed time or number of trials but not categories based on performance, it remains unclear how and when areas implicated in learning sensory-motor associations actually participate in the process. The current experiment used functional magnetic resonance imaging to examine changes in brain activation when participants learned to associate an arbitrarily located visual cue with a finger movement. Associative trials were categorized as incorrect, first correct, or subsequent correct. Participants also performed a spatially compatible visual-motor control task. A group analysis revealed four major findings addressing the behavioral processes occurring during forming and rehearsing visual-motor rules. First, brain networks related to processing associative information, through initial learning to rehearsal, yielded more activation in a myriad of neocortical structures than did a simple motor task. Second, we revealed frontal and parietal areas that differentially processed errors and correct responses. Third, we found frontal-parietal networks that seemed to mediate the transition of learning to rehearsing arbitrary visual-motor associations and that this activation exhibited dynamic characteristics. Last, we found a frontal-parietal network that appeared to have a key role in expressing the learned sensory-motor association. The current results provide a foundation for understanding how neocortical structures participate in the various behavioral processes that combine to form and consolidate novel and arbitrary sensory-motor associations.
\end{abstract}

Key words: arbitrary associative visual motor learning; evaluation; event-related functional MRI; neocortex; voluntary hand movements; trial-and-error learning

\section{Introduction}

Relationships between sensory and motor events can be learned, although the events may have no prior association. For example, although most drivers know how to respond to a traffic signal, the sensory-motor rules governing these relationships require learning, because no intrinsic association exists between a traffic signal color or its spatial position and the appropriate movements to modify the speed of an automobile. These arbitrary cue-response associations become learned through experience, commonly by trial and error.

Converging evidence suggests that several brain regions participate in the formation and rehearsal of arbitrary visual-motor associations. Neuropsychological and lesion studies indicate that damage to prefrontal cortex (PFC), premotor area (PMA), and medial temporal lobe disrupts learning and retrieving visualmotor associations (Halsband and Passingham, 1982, 1985; Pet-

Received April 4, 2003; revised Aug. 22, 2003; accepted Sept. 24, 2003.

This work was supported by grants from the National Institutes of Health (R01-AG10634, R01-NS35376, and K02-01978) and the James S. McDonnell Foundation to J.N.S. We thank the magnetic resonance research team and especially Dr. R. R. Edelman (then of Department of Radiology, Beth Israel-Deaconess Medical Center, Harvard Medical School, Boston, MA) for providing necessary support and facility access for the magnetic resonance imaging work.

Correspondence should be addressed to either of the following: Dr. James C. Eliassen, Department of Neuroscience, Box 1953, Brown Medical School, Providence, RI 02912, E-mail: James_Eliassen@Brown.edu; or Dr. Jerome N. Sanes, Department of Neuroscience, Box 1953, Brown Medical School, Providence, RI 02912, E-mail: Jerome_Sanes@Brown.edu.

Copyright $\odot 2003$ Society for Neuroscience $\quad$ 0270-6474/03/2310540-08\$15.00/0 rides, 1982, 1985, 1990, 1997; Passingham, 1985; Halsband and Freund, 1990; Murray and Wise, 1996; Gaffan and Parker, 1998). Observations of correlations between neuronal activity and learning (Mitz et al., 1991; Chen and Wise, 1995a,b, 1997; Asaad et al., 1998; Inase et al., 2001) also support a role for PFC, PMA, the supplementary eye fields, and globus pallidus in associative processing. Similarly, neuroimaging results suggest that PFC, PMA, parietal regions, and basal ganglia (Deiber et al., 1997; Toni and Passingham, 1999; Poldrack et al., 2001; Toni et al., 2001b) have roles in forming and rehearsing arbitrary visual-motor associations.

One issue not entirely resolved by lesion, neural recording, or neuroimaging work concerns how participating brain structures contribute to the stages of learning, rehearsing, and retrieving visual-motor associations, especially for spatial visual-motor associations. Previous studies have expressed the relationship between brain activity and learning as a function of elapsed time, not according to behavioral events relevant to trial-and-error learning such as correct or incorrect performance. Previous work, therefore, demonstrates time-dependent changes specific to learning in PMA, supplementary eye fields and frontal eye fields (Mitz et al., 1991; Chen and Wise, 1995a,b), PFC, basal ganglia, and parietal lobe (Deiber et al., 1997; Toni and Passingham, 1999; Poldrack et al., 2001; Toni et al., 2001a,b). However, the results of these studies do not explicitly resolve how each area participates at various stages of forming and rehearsing arbitrary associations. Because a cingulate-prefrontal network becomes 
engaged during error processing (Carter et al., 1998; Gehring and Knight, 2000) and performance improvements (Strange et al., 2001), the same network might participate in the initial formation of spatial sensory-motor rules. Similarly, structures exhibiting a late time-dependent increase in activation, such as the basal ganglia (Toni et al., 2001b), might also participate in retrieval or consolidation processes. Last, because nearly all visual-motor associations using arm movements have spatial characteristics, we hypothesized that learning spatial rules will engage frontal and parietal structures mediating visual-spatial behavior (Wise et al., 1997). By relating brain activation to actual performance, the current study addresses how different brain networks participate in the various stages of forming and rehearsing arbitrary visualmotor associations. Portions of this work have been published previously in abstract form (Eliassen et al., 1999, 2001).

\section{Materials and Methods}

Participants. Twelve healthy normal adults (age, $20-28$ years; $22.7 \pm 2.5$, mean $\pm \mathrm{SD}$; six females and six males) were recruited from the Brown University community. All participants gave written informed consent according to established institutional guidelines, and the work had ethical approval from both Brown University and Beth Israel-Deaconess Medical Center. Ten participants were right-handed (mean, 16; range, $6-20$ ), and two (males) were left-handed (scores, -11 and -18 ), as determined by a modified Edinburgh handedness survey (Oldfield, 1971), in which scores could range from -20 (left-handed) to 20 (righthanded). All participants continued in the study after being screened successfully for the lack of bodily ferromagnetic objects, and they received modest monetary compensation for participation.

Behavioral apparatus. Once situated in the magnetic resonance (MR) system, participants viewed a projection screen at the foot of the bed through an angled mirror mounted atop the head coil and adjusted to center the image of the screen in the participant's visual field. The screen was $\sim 4.5 \mathrm{~m}$ from a participant's eyes, and the entire visual display subtended $\sim 14^{\circ}$ horizontal by $11^{\circ}$ vertical. An Apple Corp. (Cupertino, CA) Macintosh PowerPC 7300/180 computer generated the visual stimuli for liquid crystal display projection (nView, Newport News, VA) and acquired response data from MR-compatible electronic buttons (Neuroscan, Sterling, VA) arranged in a linear array and housed in a response box via a digital data acquisition card (PCI-DIO-96; National Instruments, Austin, TX) at $72 \mathrm{~Hz}$.

Behavioral procedures. Participants performed two visually cued reaction time (RT) tasks in separate blocks: either a control movement task (Fig. $1 A$ ) or a visual-motor associative task (association task; Fig. 1B). Both tasks required responding by pressing one of three used buttons mounted in a response box held in the right hand. Note that a fourth button in the response box was not used (Fig. 1, the rightmost button). For the control task, the button presses had spatial compatibility with the stimulus array (Fig. 1 A, left). However, to maintain complete spatial compatibility with the response box, four stimulus positions always appeared on the display but only the three leftmost stimulus positions and buttons were used in the experiment. In the association task, the six visual stimulus positions were arranged hexagonally (Fig. $1 B$ ) about the fixation point and had an arbitrary spatial mapping with the three leftmost buttons. Two stimulus positions were mapped to each button; with this arrangement, pilot behavioral testing indicated that participants could learn six associations within the time limits of a typical MR imaging (MRI) protocol.

For both tasks, a trial began with illumination of one stimulus position (one of three in the control task and one of six in the associative task). Participants then responded as quickly as possible by pushing a single button with the index, middle, or ring finger. Participants needed to respond within $2.25 \mathrm{sec}$ after stimulus presentation, and a feedback signal appeared $\sim 2 \mathrm{sec}$ after trial onset at the location of the fixation point (Fig. $1 A, B$, right). A plus sign indicated a correct response, and a minus sign indicated an incorrect response. Across trials, the feedback signal appeared at variable times after stimulus occurrence, depending on the RT

\section{A}
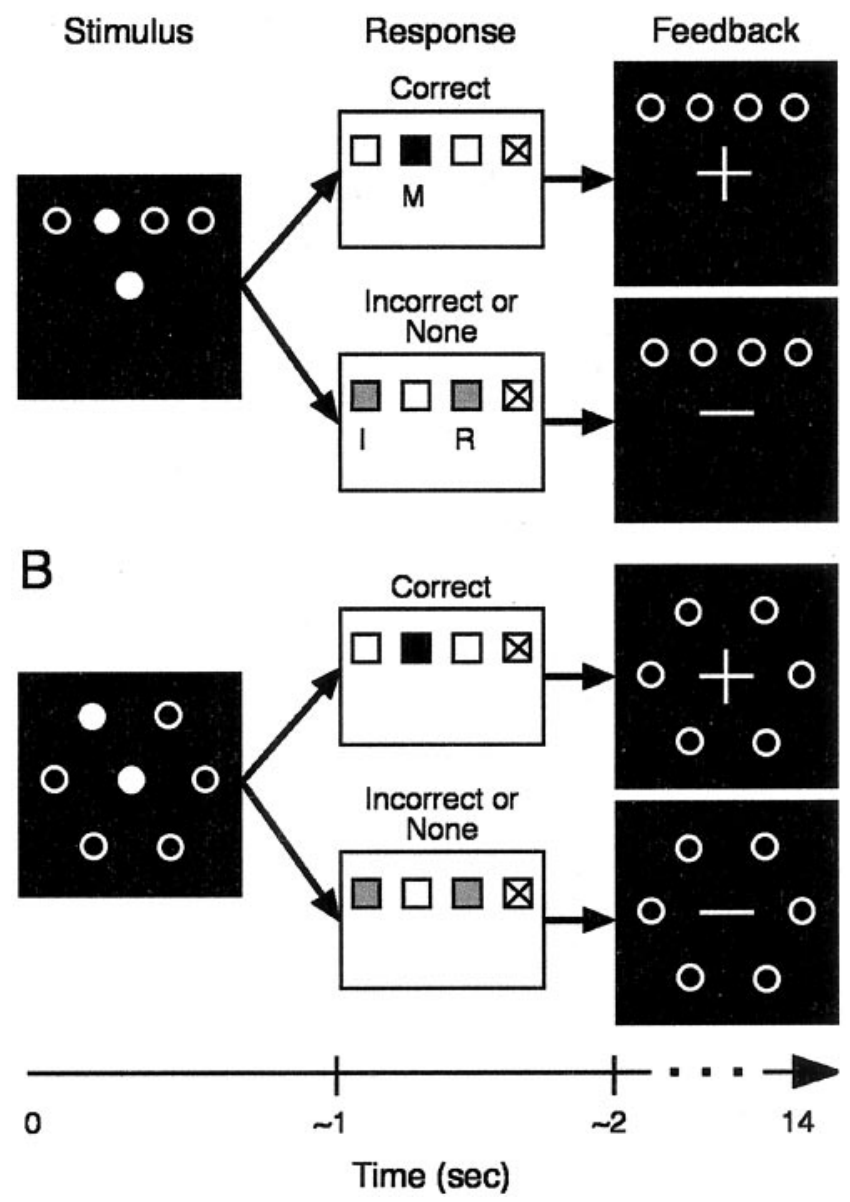

Figure 1. Schematic of the two behavioral tasks. $A$, Control task. $B$, Associative learning task. The top panels of $A$ and $B$ depict the events of a correct trial, and the bottom panels show incorrect trials. The black squares represent the screen viewed by participants, and the white rectangles represent the response box. In the examples, the response button for the middle finger $(M)$ is correct, whereas the buttons for the index $(I)$ and ring $(R)$ fingers are incorrect. The small finger button (marked with an X) was not used, although that finger rested on a button. Letters and markings are for description and did not appear on the response box. Events of a single trial for the control and association learning tasks include stimulus presentation at $0 \mathrm{sec}$ (left), followed by a response at $\sim 1 \sec$ (middle), and feedback presentation at $\sim 2 \sec$ (right).

for each trial. This was attributable to limitations in the stimulus presentation software. For RTs $>750 \mathrm{msec}$, feedback was delayed until 1200 msec after the response. For RTs between 750 and $1500 \mathrm{msec}$, we delayed feedback $600 \mathrm{msec}$. For RTs between 1500 and $2250 \mathrm{msec}$, we delayed feedback by $300 \mathrm{msec}$. Negative feedback always appeared $2250 \mathrm{msec}$ after the beginning of a trial, whereas positive feedback could occur between 1200 and $2550 \mathrm{msec}$. The feedback signal remained on for the remainder of the entire trial, which totaled $14 \mathrm{sec}$.

Before acquisition of MR images but after being positioned inside the MR system, participants received instructions and practiced the control task and a training version of the association task for $\sim 5$ min. Participants received instructions to maintain fixation throughout the experiment on the center point of the visual display. Although fixation was not monitored during the experiment, participants had to maintain fixation to perform the task adequately and also not to miss trials. We found few instances of nonresponding, indicating that participants most likely maintained fixation.

During MRI, each task was presented in 88 sec trial blocks comprising an instruction stimulus and six task trials. The experiment consisted of three trial blocks of the control task, followed by nine trial blocks of the learning task, followed by three trial blocks of the control task (Fig. $2 \mathrm{~A}$ ). 
A
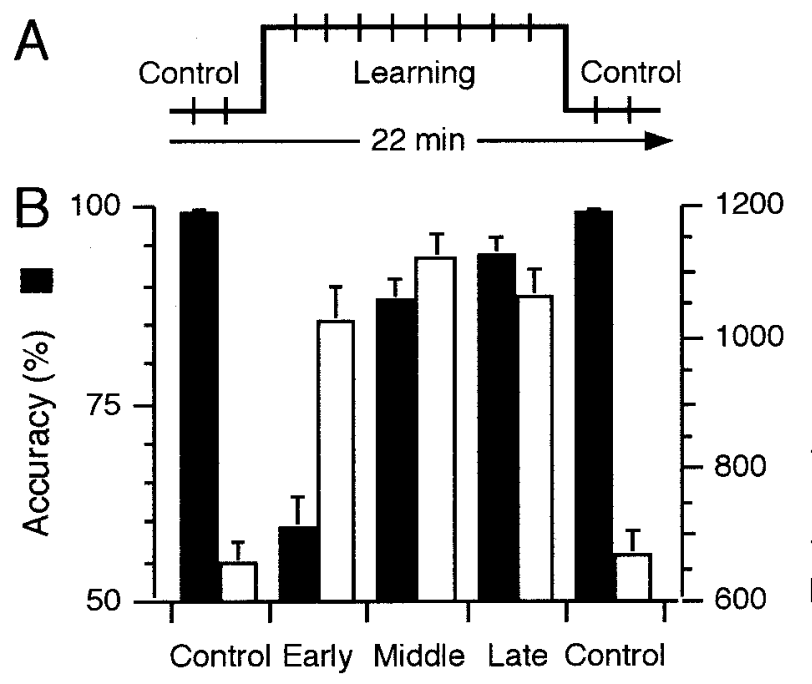

Figure 2. Experimental time line and accuracy and RT data plotted by epoch. $A$, Schematic of experimental flow indicating the flanking of learning trials by movement control trials. The vertical hash marks indicate trial blocks for the two tasks, which require a total of $22 \mathrm{~min} . B$, Accuracy and RT data for each epoch (mean of median RT \pm SEM). Accuracy data $(\square)$ are plotted with reference to the left axis. RT data $(\square)$ are plotted with reference to the right axis. For additional details, see Materials and Methods for $A$ and Results for $B$.

One participant received only two control trial blocks at the end. Hereafter, a group of three trial blocks is referred to as an epoch. Thus, the entire experiment comprised an early and late control task epoch and three association epochs (Fig. $2 B$ ). Functional MR images were acquired continuously during the performance of all epochs. At the beginning of each trial block, an instruction word, either "Move" for the control task or "Learn" for the association task, appeared for $4 \mathrm{sec}$, followed by the instruction and feedback. As noted, a single trial lasted $14 \mathrm{sec}$. For the control task, each of the three stimulus locations was presented twice per trial block, and for the association task, each of the six stimulus locations was presented once per trial block, with randomization according to Latin Squares.

Magnetic resonance imaging. A Siemens Vision Magnetom MRI system operating at $1.5 \mathrm{~T}$ and equipped for echo-planar imaging acquired the anatomical and functional MR images. Participants lay supine inside the magnet bore with the head resting inside a circularly polarized quadrature head coil used for radiofrequency transmission and reception. Cushioning minimized head movement. After shimming the standing magnetic field, we acquired a high-resolution three-dimensional anatomical data set consisting of $1601 \mathrm{~mm}$ sagittal slices [magnetization prepared rapid acquisition gradient echo sequence; repetition time (TR), $10 \mathrm{msec}$; echo time (TE), $64 \mathrm{msec}$; inversion time, $20 \mathrm{msec} ; 1 \mathrm{~mm}$ isotropic voxels; $256 \mathrm{~mm}$ field of view]. We then acquired axially oriented $\mathrm{T} 2^{\star}$-weighted gradient echo-planar images using the blood oxygenation level-dependent (BOLD) contrast mechanism (Kwong et al., 1992) with a TE of $50 \mathrm{msec}$ and a TR of $1 \mathrm{sec}$. Using a $192 \mathrm{~mm}$ field of view and an image matrix of $64 \times 64$, eight 7 -mm-thick slices were sampled from the superior convexity of the brain to the anterior terminus of the Sylvian fissure (see Fig. 4) to capture the dynamics of the event-related response in superior cortical sites, particularly in the parietal lobe. BOLD MR data were obtained continuously throughout the behavioral experiment via a series of $1588 \mathrm{sec}$ "measurements," each associated with a six-trial blocks. We acquired a total of 1320 BOLD volumes from each participant, except one who performed 14 trial blocks and obtained 1176 volumes.

Data analysis: behavior. The accuracy and RT data were segmented into the five task epochs. The number of correct responses (of a possible 18) was tabulated for each participant within an epoch, and an accuracy score and median RT were assessed with linear regression (JMP; SAS Institute, Cary, NC) using an $\alpha=0.05$. In addition, we assessed speed-accuracy tradeoffs during learning by analyzing median RTs by epoch (Fig. 2).

The epoch median RT (and functional MRI data) and the time to feedback occurrence were also segmented into nine categories, one each for the early and late control tasks and seven for the association task. We based the seven behavioral categories for the association task on the performance with each stimulus-response pair: (1) incorrect trials ("X"), for stimulus-response pairs never performed correctly; (2) trials performed correctly for the first time ("L1"); (3) trials performed correctly for the second and third time ("L23"); (4) trials performed correctly for the fourth and fifth time ("L45"); (5) trials performed correctly for the sixth, seventh, eighth, and ninth time ("L6+"); (6) trials performed incorrectly after being correct at least once ("Missed"); and (7) trials performed correctly immediately after a Missed trial ("Corrected"). For trials that followed corrected trials, the next appropriate L assignment was applied. The current procedures yielded too few missed and corrected for subsequent functional MRI analyses (group median of 3 missed and 2.5 corrected). Additionally and across the group, participants committed eight late responses. Trials categorized into missed, corrected, or late were excluded from additional analysis.

Data analysis: MRI. We used a voxel-by-voxel-based statistical procedure to identify significant activation using Analysis of Functional NeuroImages (AFNI; Medical College of Wisconsin; National Institutes of Health: http://afni.nimh.nih.gov/afni/index.shtml) (Cox, 1996; Cox and Hyde, 1997). We removed 60 volumes, four at the beginning of each measurement to eliminate T1 saturation effect. The high-resolution T1 and BOLD image sets were coregistered and normalized to the standardized space of Talairach and Tournoux (1988).

MRI signal processing. The analytical approach followed an eventrelated strategy. The BOLD data from each participant were motion corrected offline using a six-parameter rigid body transformation (Cox and Jesmanowicz, 1999), with the 98th BOLD volume as the reference. The BOLD images were then normalized to standard space with tools in AFNI and resampled to a resolution of $3 \times 3 \times 3 \mathrm{~mm}$ and smoothed with a $6 \times 6 \times 6 \mathrm{~mm}$ Gaussian kernel. We used deconvolution procedures to estimate the hemodynamic response during behavioral task performance. Three separate deconvolution calculations were performed for each participant, corresponding to the early control task epoch, the association task epochs, and the late control task epoch. The deconvolution reference functions were based on task performance and the motion correction parameters. The procedure estimated the average BOLD signal, linear drift, and the contributions of the behaviorally defined reference functions. No additional low-frequency components were removed. The nine behavioral categories described above were used to construct reference functions that indicated the beginning of a trial for each category. The deconvolution estimated 13 coefficients, one at each of 13 lags after stimulus presentation $(0-12 \mathrm{sec})$. These coefficients were then normalized to the average BOLD to derive a percentage change signal; time courses were also normalized to the average of the second and third time points in the trial to remove any residual signal offsets present in the event-related hemodynamic response.

MRI statistical analysis. The baseline and temporally normalized activation maps were submitted to two-factor ANOVA using trial category (fixed-effect) and participant (random-effect) as dependent variables. Mean statistical maps across time (3-12 sec; poststimulus) for the seven associative categories were contrasted to test different hypotheses, as described in Results. We also performed a linear regression analysis across the learning trial categories to assess the transition from initial to later correct responding. We applied multiple thresholds to the statistical maps. First, we restricted the analysis to voxels acquired from all participants (see Fig. 4A). Second, the mean statistical activation maps were used to create a mask of "active regions" (Fig. $4 B$ ), which restricted the volume of analysis. The active regions comprised those voxels from the mean maps with significant activation in at least two time points in one of the nine behavioral categories. The intersection of the active regions from the motor control task and the associative learning task indicated the degree of activation overlap between the tasks (Fig. 4C). We used a voxel level threshold of $p \leq 0.005(t \geq 2.9)$ and a cluster level threshold of $p<$ 0.05 ( 22 contiguous voxels). Additionally, we applied a threshold requiring cluster level activation for two adjacent time points. We used the Brodmann area (BA) localizer tool implemented in AFNI to assign activation to specific brain regions. 


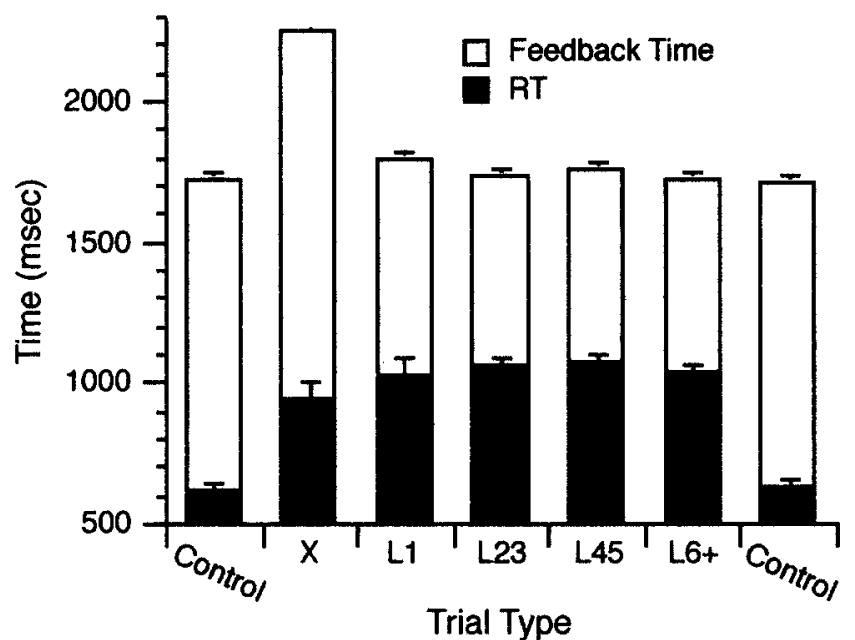

Figure 3. RT and feedback appearance time (mean of medians \pm SEM) plotted by behavioral task category. $\square$, RT for the various task categories; $\square$, time at which feedback appears. Note the rough correspondence between RTs and feedback times, except for the initially incorrect $(X)$ and the movement control trials.

\section{Results}

\section{Behavior}

As expected, participants performed more accurately for the movement control than for the association task (Fig. 2B). Progressive increases in accuracy with repeated performance indicated that learning occurred during the association task (Fig. 2 B). A regression analysis with accuracy as the dependent variable yielded a significant whole-model effect $(p<0.0001)$ accounting for $87 \%$ of the variance $\left(R^{2}=0.87\right)$. Accuracy differed significantly between the control epochs (each 99\% correct) and the association task epochs (59, 88, and 94\% correct; $p<0.0001)$. Accuracy improved across the three association task epochs, differing significantly between the first association task epoch and the second and third epochs $(p<0.0001)$ and between the second and third association task epochs $(p<0.05)$. As a group, all the participants learned the correct associations before the end of the associative task epochs.

Analysis of RT by epoch (Fig. 2B) and category (Fig. 3) indicated learning-related changes. A regression analysis of median RT by epoch (Fig. $2 B, \square$ ) yielded a significant whole-model effect $(p<0.0001)$, that accounted for $87 \%$ of the variance $\left(R^{2}=\right.$ $0.87)$. RTs increased significantly from the early to middle epoch of the association task $(p<0.05)$ but exhibited only a trend toward decrease from the middle to late epoch of the association task $(p<0.1)$. Because accuracy also increased significantly from the early to middle association task epochs, this may signify a speed-accuracy tradeoff. Regression analysis of median RTs by performance category (Fig. 3, $\mathbf{\square}$ ) yielded a significant wholemodel effect $(p<0.0001)$ accounting for $73 \%$ of the variance $\left(R^{2}=0.73\right)$. Median RTs differed significantly between the movement control and association tasks $(p<0.0001)$. RTs obtained during incorrect trials differed significantly from RTs obtained during correctly performed trials (L1 and rehearsal combined; $p<0.05, \mathrm{X}$ vs all L trials in Fig. 3), but RTs on L1 trials did not differ from RT during rehearsal trials $(p>0.4)$. The number of trials for each category varied (mean \pm SEM): early and late control task epochs, 18 trials each: X, $6.8 \pm 2$ trials (range, $4-11$ ); L1, 6 trials; L23, $11.6 \pm 0.8$ trials (range, 10-12); L45, $10.8 \pm 1.3$ trials (range, 8-12); L6+, $12.3 \pm 4.5$ trials (range, $5-19$ ).

We found small, significant differences in feedback times

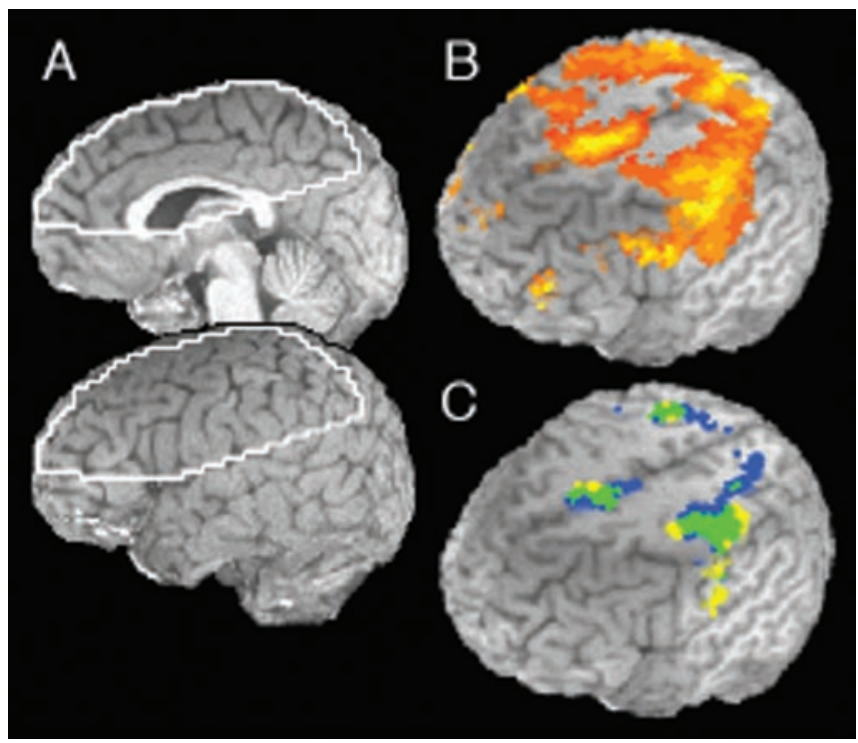

Figure 4. Imaging coverage, active regions, and activation overlap. $A$, The white outline shows the brain regions covered by the imaging sequence, for which functional MRI data were obtained in all 12 participants. Data analysis was restricted to these commonly imaged regions. $B$, Active regions of neocortex (see details in Materials and Methods). The color scale ranges from 0 to $0.6 \%$ increased activation (red to yellow). C, Activation overlap between associative task and control task activation. The overlap is descriptive, not the result of an inferential statistical test. Yellow, Activated during the movement control task; blue, activated during the associative task; green, activated for both the control and associative tasks. Renderings cut away superior to $+48 \mathrm{~mm}(\mathrm{Z})$ and left of $+58 \mathrm{~mm}(\mathrm{X})$. Activation is overlaid on a single participant's high-resolution, T1-weighted image.

among the behavioral task categories (Fig. 3, $\square$ ). The regression analysis on feedback time yielded a significant whole-model effect $(p<0.0001)$ accounting for $90 \%$ of the variance $\left(R^{2}=0.9\right)$. Incorrect feedback always appeared at $2250 \mathrm{msec}$. Incorrect feedback time differed significantly from correct trials $(p<0.0001)$. Rehearsal trial feedback times were not significantly different from control $(p>0.3)$. First time correct feedback times, however, were significantly longer than rehearsal trials times $(p<0.05)$.

\section{Brain activation}

Brain areas became activated during both the control and the association tasks (Fig. $4 B, C$ ). Figure $4 B$ shows the brain space constituting the active regions used to restrict the functional analyses (see Materials and Methods). Figure $4 C$ shows overlap of regional activation (in green) during both control task epochs and each association task category. The activated regions included the left Rolandic region; a cluster overlapping medial BAs 6,24 , and 32; and the left and right superior parietal lobule (BA 7). This depiction does not reflect results derived from a statistical conjunction analysis (Price and Friston, 1997) but simply shows common activation for the different conditions. Different stages of association learning and rehearsal modulated activation in parietal and frontal cortex. The comparisons between early and mid-rehearsal (L23 vs L45) and that between early and late control task epochs did not reveal any significant activation, but all other statistical contrasts yielded significant activation (Table 1).

A contrast between trials performed incorrectly at the outset of associative learning with correctly performed trials could reveal brain networks concerned with processing errors. Several brain areas exhibited greater activation for incorrectly than for correctly performed trials (Fig. 5), including the anterior cingu- 
Table 1. Active cluster attributes

\begin{tabular}{|c|c|c|c|c|c|}
\hline \multirow[b]{2}{*}{ Comparison region (BA) } & \multicolumn{3}{|c|}{ Coordinates } & \multirow[b]{2}{*}{ Volume $(\mu l)$} & \multirow[b]{2}{*}{ Time (sec) } \\
\hline & $X$ & Y & $Z$ & & \\
\hline \multicolumn{6}{|l|}{ Errors > correct (Fig. 5) } \\
\hline Cingulate gyrus (32) $B$ & -3 & -24 & 33 & 6075 & $3,6-12$ \\
\hline R SFG (9) & -16 & -48 & 26 & 270 & $9,11-12$ \\
\hline R IFG/MFG (9/46) & -40 & -24 & 26 & 2808 & $3,7,9-12$ \\
\hline LIFG/MFG (9) D & 44 & -10 & 31 & 1377 & $3,7,9-12$ \\
\hline R MFG (6) & -22 & -3 & 48 & 297 & 10,12 \\
\hline R MFG (6) & -25 & 10 & 40 & 1188 & $3,10-12$ \\
\hline L MFG (9/46) & 39 & -29 & 27 & 297 & $9-12$ \\
\hline L Precentral gyrus (6) E & 49 & 9 & 38 & 1026 & $7-11$ \\
\hline R IPL/postcentral gyrus (40/2) & -36 & 31 & 44 & 3537 & $9-12$ \\
\hline${ }^{*}$ L Pre-/postcentral gyrus (4/3) C & 35 & 25 & 63 & 2079 & $6-8,10-11$ \\
\hline RIPL/SPL (40/7) & -36 & 55 & 47 & 1080 & $11-12$ \\
\hline LIPL (40) F & 38 & 43 & 43 & 1998 & $8-12$ \\
\hline LSPL/precuneus (7) & 15 & 61 & 50 & 1404 & $8-12$ \\
\hline *Cingulate gyrus (23) & 1 & 24 & 28 & 810 & $4-10,12$ \\
\hline \multicolumn{6}{|l|}{ L1 > rehearsal (Fig. 6) } \\
\hline R medial SFG (9) $B$ & -4 & -48 & 29 & 351 & $7-8$ \\
\hline R IFG $(44 / 45) F$ & -50 & -16 & 17 & 999 & 7 \\
\hline LIFG (45)E & 41 & -20 & 17 & 810 & 8 \\
\hline${ }^{*} \mathrm{~L}$ Cingulate gyrus (24) C & 6 & 11 & 41 & 513 & $10-11$ \\
\hline${ }^{*} \mathrm{LIPL}(40) D$ & 46 & 40 & 47 & 189 & $5-6$ \\
\hline \multicolumn{6}{|l|}{ Early > late rehearsal (Fig. 7) } \\
\hline R Postcentral gyrus (2/3) & -46 & 24 & 49 & 729 & $6-10$ \\
\hline L Precuneus (7) C & 10 & 61 & 37 & 594 & $6-7,10-11$ \\
\hline L Precuneus (7) & 2 & 53 & 49 & 486 & $6-10$ \\
\hline L Anterior thalamus $B$ & 7 & 9 & 10 & 594 & $7,9-11$ \\
\hline \multicolumn{6}{|l|}{ Rehearsal > control (Fig. 8) } \\
\hline LIFG/MFG (9) C & 44 & -9 & 31 & 594 & 4 \\
\hline L Precentral gyrus (6) $B$ & 27 & 16 & 55 & 594 & 5 \\
\hline LIPL (7) D & 26 & 62 & 39 & 162 & $4-5$ \\
\hline
\end{tabular}

An asterisk $\left(^{*}\right)$ indicates an activation pattern reverse from that indicated. Italic capital letters after the area refer to appropriate panels in Figs. 5-8. Coordinates represent the cluster center-of-mass, with $+X=$ left of anterior commissure $(A C),+Y=$ posterior to $A C$, and $+Z=$ superior to $A C$. Cluster volume in microliters; one voxel $=27$ $\mu \mathrm{l}$. Time (sec) indicates at which time a cluster exhibited different activation between conditions. MFG, Middle frontal gyrus.

late cortex and several other frontal and parietal areas bilaterally (Table 1). These areas commonly exhibited activation for correct trials (Fig. $5 B, D-F$ ), but greater activation for incorrectly performed trials emerged $6-7$ sec after trial onset and generally persisted until the end of the analysis period. Two brain regions exhibited greater activation for correctly than for incorrectly performed trials, a superior left precentral gyrus region and the left middle cingulated gyrus; the timing of the activation difference in these areas paralleled that of the other regions. Thus, activation differences across brain regions, regardless of their sign, emerged soon after the evoked hemodynamic response began and persisted until the end of a trial.

Areas that exhibit activation differences between the first correct association trial (L1) and subsequent correct trials (L23L6+) could differentiate areas that participate in initial acquisition of the associative response from those active in subsequent retrieval of learned associations. We found two patterns of activation when comparing L1 with L23-L6+ trials (Fig. 6). In the right medial superior frontal gyrus (SFG; BA 9) and the left and right inferior frontal gyri (IFG; BA 44/45), L1 trials yielded higher activation than for rehearsal trials. This activation could have consistency with encoding processes related to the correct association or recognition and evaluation of correct performance. In contrast, activation in the left inferior parietal lobule (IPL) and left cingulate cortex showed greater activation for rehearsal (L23L6+ ) than for L1 trials, which could have consistency with establishing representations of the learned associations. In addition to having different activation for L1 and rehearsal trials (Table 1),
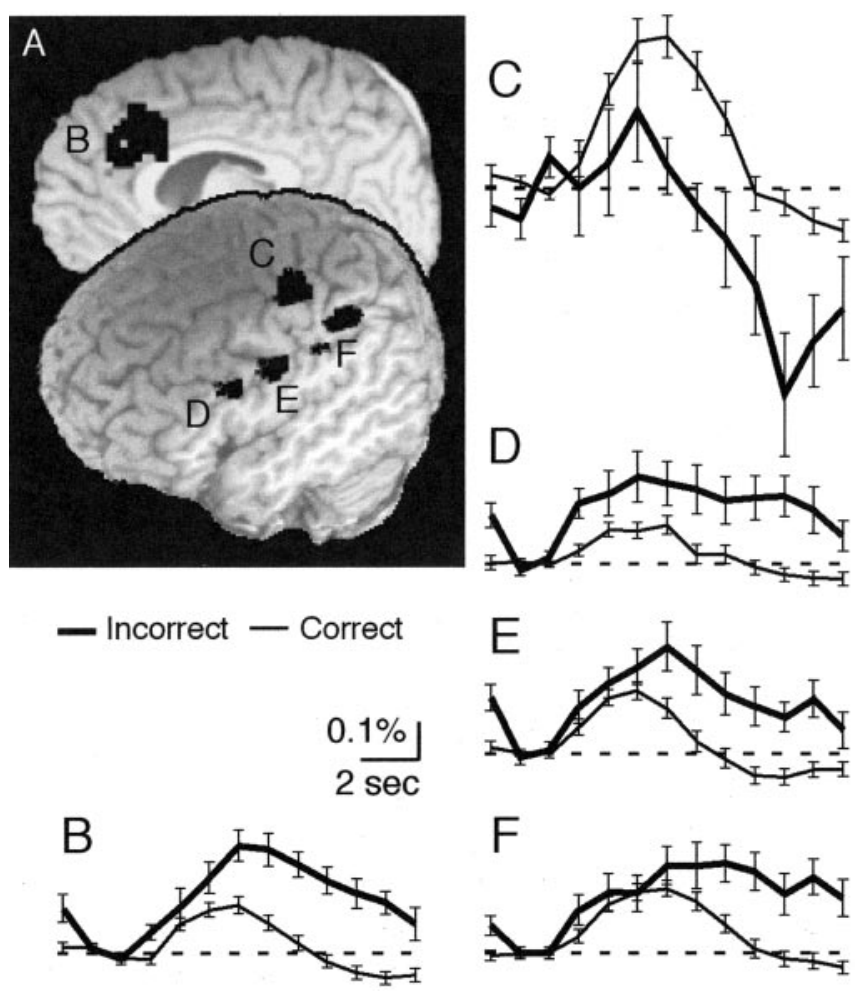

Figure 5. Brain responses for errors compared with correct performance. $A$, Activation clusters occurred bilaterally in the anterior cingulate $(B)$, left SPG $(C)$, left middle/IFG $(D)$, left ventral precentral gyrus $(E)$, and left IPL $(F)$. Activation also appeared in other locations (Table 1). $B-F$, Time courses of functional MRI signal for the anterior cingulated $(B)$, left precentral gyrus $(C)$, left inferior/middle frontal gyrus $(D)$, left ventral precentral gyrus $(E)$, and left IPL $(F)$. Cluster coordinates and times of significant differential activation are shown in Table 1.

the right medial SFG and the right and left IFG, alone among brain regions, also exhibited linearly decreasing activation as performance progressed across trials performed correctly [i.e., L1 though L6+ (Fig. 6)]. For the right medial SFG, increased activation occurred at 7 and $8 \mathrm{sec}$ after stimulus onset, and for the right IFG, the enhanced activation occurred 7, 8, and 9 sec after stimulus, whereas the left IFG showed increased activation only at $8 \mathrm{sec}$ after stimulus onset.

We also found evidence of consolidation of the sensory-motor association by comparing functional MRI signals obtained in the early (L23, L45) and later (L6+) stages of rehearsal. Four brain regions exhibited a decreasing activation pattern from early to late rehearsal (Fig. 7); these regions included the left anterior thalamus, the right postcentral gyrus, and two separable clusters located in the left precuneus.

Finally, we compared the functional MR signal obtained for rehearsal (L23-L6+) and the nonassociative movement control task to determine regions that might participate in expression of the learned associations. Brain regions that store and retrieve associative information might exhibit more activation during rehearsal of visual-motor associations in comparison with a direct pointing task. We found that rehearsing the visual-motor association yielded greater activation than the nonassociative control task in three regions (Fig. 8). The left IPL exhibited significantly greater activation 4 and $5 \mathrm{sec}$ after stimulus presentation. The left IFG (BA 45) exhibited increased activation at $4 \mathrm{sec}$ after stimulus, whereas a cluster encompassing the left precentral/middle frontal gyrus (BA 6) showed increased activation $5 \mathrm{sec}$ after stimulus onset. 

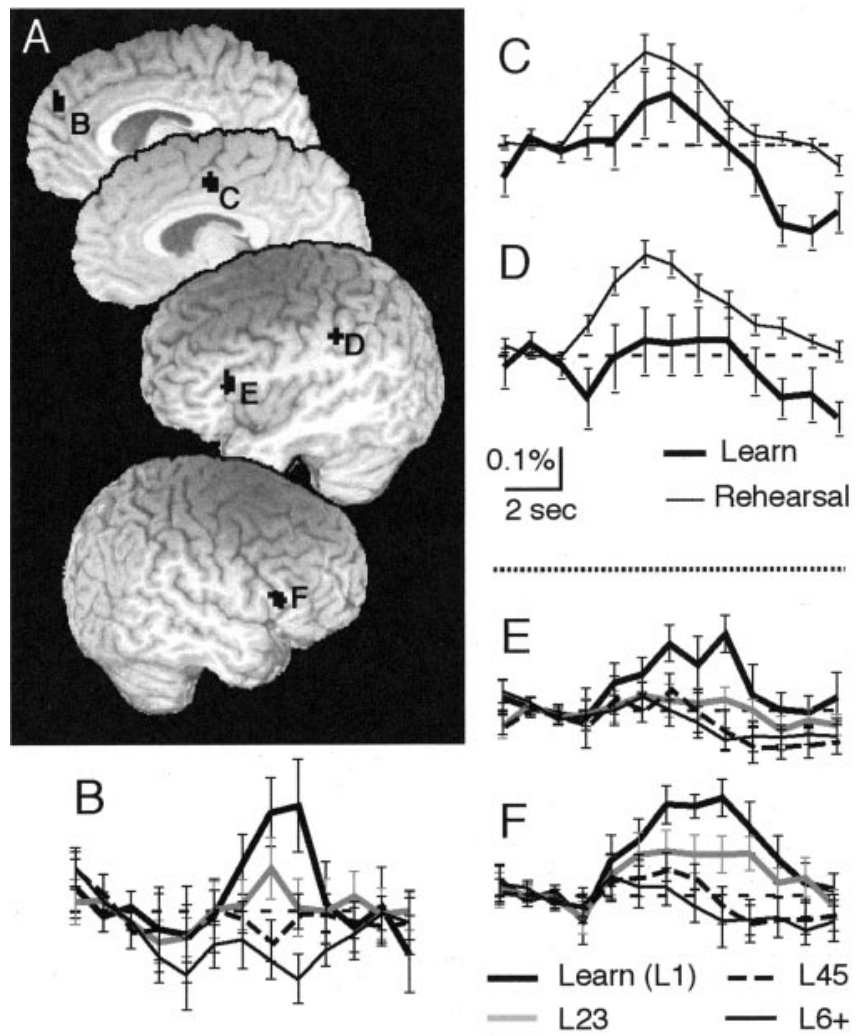

Figure 6. Brain activation for the first correct association trial compared with rehearsal. $A$, Activation clusters occurred in the right medial SFG $(B)$, the left cingulate gyrus $(C)$, left IPL $(D)$, and left and right IFG $(E, F)$. Clusters $B, E$, and $F$ showed a statistically significant linear decrement in activation from the first through subsequent correct trials. These same clusters also showed a statistically significant difference in activation between the first and subsequent correct trials (compare regression coordinates below with ANOVA results shown in Table 1). The activation time courses are shown in $B, E$, and $F$. $B-F$, Time courses for right medial SFG (B; significant from 7-8 sec; $X=-3, Y=-48, Z=30 ; 12$ voxels), the left cingulate $(C)$, the left superior parietal lobule $(D)$, left IFG ( $E$; significant at $8 \mathrm{sec} ; X=40, Y=-21, Z=17 ; 26$ voxels), and the right IFG (F; significant from 7-9 sec; $X=-51, Y=-18, Z=19 ; 18$ voxels). Other coordinates and times of significant activation are shown in Table 1.

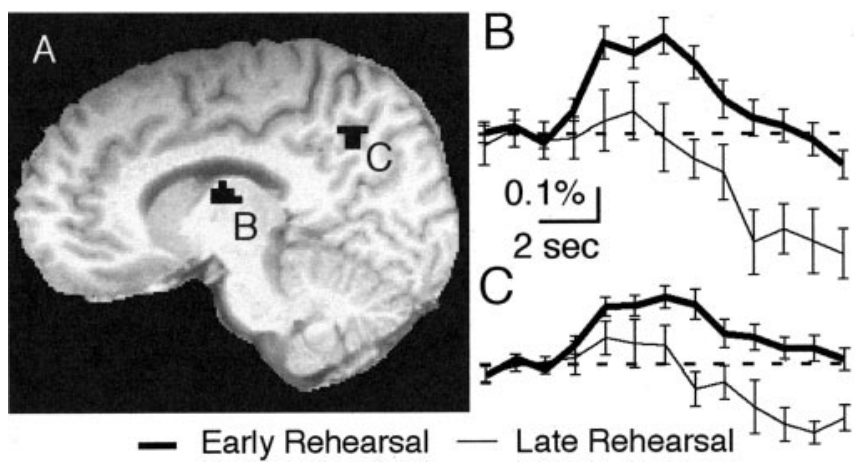

Figure 7. Functional MRI signal comparing early and late rehearsal. $A$, Activation clusters appeared in thalamus and precuneus. $B, C$, Time courses of activation for the left thalamus $(B)$ and left precuneus ( $C$ ). Cluster coordinates and times of significant activation are shown in Table 1.

\section{Discussion}

Considerable evidence exists for cerebral cortical and subcortical roles in learning and rehearsing arbitrary relationships between visual inputs and voluntary movements (Mitz et al., 1991; Toni and Passingham, 1999; Toni et al., 2001b). The available data
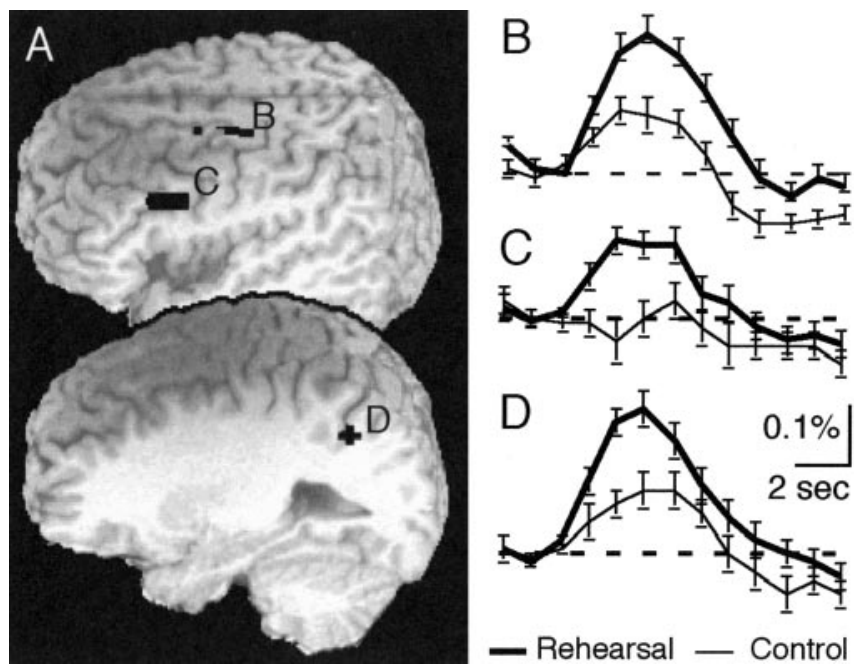

Figure 8. Activation comparing rehearsing the association and movement. $A$, Extracted clusters occurred in the dorsal precentral gyrus $(B)$, left inferior frontal sulcus $(C)$, and IPL $(D)$. $B-D$, Time courses of activation in the left inferior frontal sulcus $(B)$, left dorsal precentral gyrus $(C)$, and IPL $(D)$. Cluster coordinates and times of significant activation are shown in Table 1.

mostly describe learning-related changes in neural spiking or brain activation without noting whether the changing patterns reflect cognitive operations necessary for trial-and-error learning of the arbitrary associations. This experiment addressed the behavioral processes underlying formation and rehearsal of visualmotor rules. The data indicated frontal and parietal activation generally related to performing the associative task and specifically to differences processing errors and correct responses, the transition from learning to rehearsal, and the expression of acquired associations. The current results provide a basis to comprehend how neocortex participates in behavioral processes for forming and consolidating rules to associate novel and arbitrary sensory and motor events. Our results have broad consistency with other studies of learning and error production.

The initial phases of trial-and-error learning primarily involve error or conflict processing. Consistent with activation in this study, error and conflict processing engage a prefrontal-anterior cingulate network (Carter et al., 1998; Gehring and Knight, 2000; Kiehl et al., 2000). The left lateral PFC activation observed here coincides with reported negative feedback activation (Monchi et al., 2001). Similar to other studies of error and response conflict (Carter et al., 2000; Monchi et al., 2001; van Veen et al., 2001), we find greater parietal activation during error commission. This activation endures well into the trial and may relate to feedback processing, although the short nearly constant interval between stimulus and feedback prevents us from evaluating whether the persistent activation relates to errors or feedback processing. Recent work in our laboratory indicates specific patterns of feedback-related activation in frontal and parietal structures (Eliassen et al., 2003).

Subsequent to initial errors, successful performance of the associative task requires forming and maintaining the correct visual-motor association. Performance evaluation and memory acquisition have key roles in these processes. Two observations suggest that medial SFG and lateral IFG activation and subsequent decrement represent performance evaluation. First, PFC activation decreases with rehearsal when feedback, which initially guides learning by providing evaluative information, becomes less consequential to performance. Second, several reports suggest that medial and lateral $\mathrm{PFC}$ regions similar to those activated here participate in evaluating internal states (Castelli et al., 2000; 
Gusnard et al., 2001; Zysset et al., 2002). Alternatively, medial and lateral PFC activation may reflect memory acquisition or encoding (Wagner et al., 1998; McDermott et al., 1999; Jessen et al., 2001), a process that in most cases occurs during the first correct trial. Our findings do not clearly discriminate between these alternatives, but, notably, memory-encoding tasks often involve stimulus evaluation: rating the pleasantness of an item (McDermott et al., 1999), determining whether a word is concrete or abstract (Wagner et al., 1998), or deciding whether an item has been observed previously (Jessen et al., 2001). We deduce from these patterns that the PFC regions activated early in learning do not likely have a continued memory function, because activation in these areas declines with continued task performance, although memory and associative functions must still be engaged to perform correctly.

It might be expected that the brain regions supporting a continued memory or associative function beyond initial acquisition would exhibit increased activation from initial correct performance to rehearsal. Activation of IPL and mid-cingulate cortex might support such cognitive processes, because activation occurs after the first correct response. The activation of the left IPL and precentral gyrus during rehearsal may also reflect expression or retrieval of learned rules. PMA and IPL activation increases from learning to rehearsal (data not shown; but X and L1 trials exhibit activation levels between rehearsal and control levels at 5-6 $\mathrm{sec})$. These data suggest a potentiation of the response in IPL, PMA, and mid-cingulate cortex during rehearsal. The spatial context of association expression may explain IPL activation, because Grafton et al. (1998) observed nearby activation during association rehearsal. Previous findings may also help clarify precentral gyrus activation. Premotor activation has been observed with the expression of arbitrary associations (Grafton et al., 1998), but not always (Deiber et al., 1997; Toni and Passingham, 1999; Toni et al., 2001b). PMA activation might be expected because lesions to PMA (putatively located in the precentral gyrus) disrupt the recall of preoperatively learned associations (Delacour et al., 1972), and changes in neural activity in the PMA are altered primarily in response to retrieval rather than to learning associations (Mitz et al., 1991). However, PMA lesions disrupt the learning of stimulus-movement associations but not stimulus-location associations (Halsband and Freund, 1990). Our observations of premotor and parietal activation during rehearsal correspond well to previous work and suggest a specific role for these areas in continued memory or associative functions beyond initial learning.

Lateral PFC BA 9 exhibits a noteworthy activation pattern. Activation in BA 9 declines from errors to correct trials and decreases from rehearsal to control task performance. The decrement in activation is particularly prominent at the late time points in the trial, whereas maintenance of activation when comparing late learning to movement occurs early in the trial. This pattern of decreasing activation with learning and sustained activation during rehearsal is consistent with tuning, processswitching, or a reduction in processing time, all hypothetical models of learning-related changes in brain activation (Poldrack et al., 1998; Poldrack, 2000). In the tuning model, the hemodynamic response becomes centered on a particular event within the trial, possibly stimulus presentation. Alternatively, like many of the brain regions exhibiting greater activation for errors, the reduction in activation late in the trial might reflect a switch in the underlying process driving activation in this brain region (e.g., a shift from a feedback-locked mode to a response-locked mode). Last, a reduction in late activation could reflect a reduction in processing time for one or more events within a trial. This expla- nation derives support from the changes in reaction and feedback times. The activation in PFC is also consistent with a role in response selection (Rowe et al., 2000), because activation is highest when the number of potential response alternatives is high and decreases once the correct response is known and the number of potential alternatives goes to zero. The continued activation of BA 9 during rehearsal may then reflect an ongoing participation in the response selection or associative process. The limitations of this experiment in distinguishing stimulus- and feedback-locked activation have prompted additional examination of this issue (Eliassen et al., 2003).

The decreases in activation observed at all of the behavioral transitions warrant additional discussion. If the observed decreases could be characterized as progressive, they might be confounded with learning effects and possibly attributable to simple passage of time, task novelty, or another incremental process. Two observations argue against such possibilities. First, four regions exhibit increases in activation at early transitions in the experiment. Second, only one region, the left IFG/MFG (BA 9) exhibits significant activation changes at more than one transition. Thus, the decreases may be unique to certain brain regions at particular behavioral transitions. Task novelty and time could, nevertheless, have confounding influences. The performance progression can only take place in a certain temporal order. Our data processing techniques normalize that progression for each participant and each cue-response pair. Although the underlying neural processes may not have a discrete nature, as we claim, they certainly must occur in the order we have specified, although the effects of time, experience, and task novelty remain intertwined to some degree and difficult to discriminate.

Similar to previous results on associative learning (Sakai et al., 1998, 1999), we found activation transitions from frontal to parietal occurring as learning progressed. The reduction of presumed frontal "executive" processes with learning and the engagement of parietal sites during rehearsal could explain such a shift (Poldrack et al., 1998; Poldrack, 2000). The simultaneous activation of PFC regions responsive to evaluation and memory encoding, noted above, could signify the integration of stimulus, response, and outcome information (Passingham et al., 2000) into an arbitrary rule for behavior (Miller, 2000), or it could signify the recruitment of cognitive control processes when the rules are not known (Miller and Cohen, 2001). Frontal activation declines as responses come to be guided by experience. Increases in parietal activation during rehearsal might be the expression of this experience, linking perceptual, motor, and outcome events from previous trials into an associative representation that guides current behavior.

Our findings suggest that in the context of arbitrary spatial visual-motor association learning, rule acquisition and rule expression selectively engage distinct brain regions. Medial and lateral inferior prefrontal regions are engaged by associative rule acquisition. Premotor and parietal areas are engaged by the behavioral expression of learned rules. In addition, brain activation changes in relation to the history of performance. Because our classification of imaging data are based on performance history rather than accuracy or response time measures, these data constitute a straightforward indication of experience-dependent modulation of brain activation.

\section{References}

Asaad W, Rainer G, Miller EK (1998) Neural activity in the primate prefrontal cortex during associative learning. Neuron 21:1399-1407. Carter CS, Braver TS, Barch DM, Botvinick MM, Noll D, Cohen JD (1998) Anterior cingulate cortex, error detection, and the online monitoring of performance. Science 280:747-749. 
Carter CS, MacDonald AW, Botvinick MM, Ross LL, Stenger VA, Noll D, Cohen JD (2000) Parsing executive processes: strategic vs. evaluative functions of the anterior cingulate cortex. Proc Natl Acad Sci USA 97:1944-1948.

Castelli F, Happé F, Frith U, Frith C (2000) Movement and mind: a functional imaging study of perception and interpretation of complex intentional movement patterns. NeuroImage 12:314-325.

Chen LL, Wise SP (1995a) Neuronal activity in the supplementary eye field during acquisition of conditional oculomotor associations. J Neurophysiol 73:1101-1121.

Chen LL, Wise SP (1995b) Supplementary eye field contrasted with the frontal eye field during acquisition of conditional oculomotor associations. J Neurophysiol 73:1122-1133.

Chen LL, Wise SP (1997) Conditional oculomotor learning: population vectors in the supplementary eye field. J Neurophysiol 78:1160-1163.

Cox RW (1996) AFNI: software for analysis and visualization of functional magnetic resonance neuroimages. Comput Biomed Res 29:162-173.

Cox RW, Hyde JS (1997) Software tools for analysis and visualization of FMRI data. NMR Biomed 10:171-178.

Cox RW, Jesmanowicz A (1999) Real-time 3D image registration for functional MRI. Magn Reson Med 42:1014-1018.

Deiber M-P, Wise SP, Honda M, Catalan MJ, Grafman J, Hallett M (1997) Frontal and parietal networks for conditional motor learning: a positron emission tomography study. J Neurophysiol 78:977-991.

Delacour J, Libouban S, McNeil M (1972) Premotor cortex and instrumental behavior in monkeys. Physiol Behav 8:299-305.

Eliassen J, Baker J, Bonynge G, Souza T, Sanes J (1999) Cortical timing and activation patterns during spatial conditional motor association learning. Soc Neurosci Abstr 25:1410.

Eliassen J, Souza T, Sanes J (2001) Distinct cortical activation for rule formation and implementation related to arbitrary spatial visual motor associations. NeuroImage 13:S1159.

Eliassen JC, Johnson MB, Sanes JN (2003) Experience dependent modulation of stimulus- and feedback-related hemodynamic responses during arbitrary association learning. Presented at the 9th International Conference on Functional Mapping of the Human Brain, New York, NY, June 19-22, 2003. Available on CD-ROM in Neuroimage, Vol 19, No 2.

Gaffan D, Parker A (1998) Memory after frontal/temporal disconnection in monkeys: conditional and non-conditional tasks, unilateral and bilateral frontal lesions. Neuropsychologia 36:259-271.

Gehring WJ, Knight RT (2000) Prefrontal-cingulate interactions in action monitoring. Nat Neurosci 3:516-520.

Grafton ST, Fagg AH, Arbib MA (1998) Dorsal premotor cortex and conditional movement selection: a PET functional mapping study. J Neurophysiol 79:1092-1097.

Gusnard DA, Akbudak E, Shulman GL, Raichle ME (2001) Medial prefrontal cortex and self-referential mental activity: relation to a default mode of brain function. Proc Natl Acad Sci USA 98:4259-4264.

Halsband U, Freund H-J (1990) Premotor cortex and conditional motor learning in man. Brain 113:207-222.

Halsband U, Passingham RE (1982) The role of premotor and parietal cortex in the direction of action. Brain Res 240:368-372.

Halsband U, Passingham RE (1985) Premotor cortex and the conditions for a movement in monkeys. Behav Brain Res 18:269-277.

Inase M, Li BM, Takashima I, Iijima T (2001) Pallidal activity is involved in visuomotor association learning in monkeys. Eur J Neurosci 14:897-901.

Jessen F, Flacke S, Granath D-O, Manka C, Scheef L, Papassotiropoulos A, Schild HH, Heun R (2001) Encoding and retrieval related cerebral activation in continuous verbal recognition. Cognit Brain Res 12:199-206.

Kiehl KA, Liddle PF, Hopfinger JB (2000) Error processing and the rostral anterior cingulate: an event-related fMRI study. Psychophysiology $37: 216-226$.

Kwong KK, Belliveau JW, Chesler DA, Goldberg IE, Weisskoff RM, Poncelet BP, Kennedy DN, Hoppel BE, Cohen MS, Turner R, Chen H-M, Brady TJ, Rosen BR (1992) Dynamic magnetic resonance imaging of human brain activity during primary sensory stimulation. Proc Natl Acad Sci USA 89:5675-5679.

McDermott KB, Ojemann JG, Petersen SE, Ollinger J, Snyder AZ, Akbudak E, Conturo T, Raichle ME (1999) Direct comparison of episodic encoding and retrieval of words: an event-related study. Memory 7:661-678.

Miller EK (2000) The prefrontal cortex and cognitive control. Nat Rev Neurosci 1:59-65.
Miller EK, Cohen JD (2001) An integrative theory of prefrontal cortex. Annu Rev Neurosci 24:167-202.

Mitz AR, Godschalk M, Wise SP (1991) Learning-dependent neuronal activity in the premotor cortex: activity during the acquisition of conditional motor associations. J Neurosci 11:1855-1872.

Monchi O, Petrides M, Petre V, Worsley K, Dagher A (2001) Wisconsin card sorting revisited: distinct neural circuits participating in different stages of the task identified by event-related functional magnetic resonance imaging. J Neurosci 21:7733-7741.

Murray EA, Wise SP (1996) Role of the hippocampus plus subjacent cortex but not amygdala in visuomotor conditional learning in rhesus monkeys. Behav Neurosci 110:1261-1270.

Oldfield RC (1971) The assessment and analysis of handedness: the Edinburgh inventory. Neuropsychologia 9:97-113.

Passingham RE (1985) Premotor cortex: sensory cues and movement. Behav Brain Res 18:175-185.

Passingham RE, Toni I, Rushworth MF (2000) Specialisation within the prefrontal cortex: the ventral prefrontal cortex and associative learning. Exp Brain Res 133:103-113.

Petrides M (1982) Motor conditional associative-learning after selective prefrontal lesions in the monkey. Behav Brain Res 5:407-413.

Petrides M (1985) Deficits in non-spatial conditional associative learning after periarcuate lesions in the monkey. Behav Brain Res 16:95-101.

Petrides M (1990) Nonspatial conditional learning impaired in patients with unilateral frontal but not unilateral temporal lobe excisions. Neuropsychologia 28:137-149.

Petrides M (1997) Visuo-motor conditional associative learning after frontal and temporal lesions in the human brain. Neuropsychologia 35:989-997.

Poldrack RA (2000) Imaging brain plasticity: conceptual and methodological issues - a theoretical review. NeuroImage 12:1-13.

Poldrack RA, Desmond JE, Glover GH, Gabrieli JD (1998) The neural basis of visual skill learning: an fMRI study of mirror reading. Cereb Cortex 8:1-10.

Poldrack RA, Clark J, Pare-Blagoev EJ, Shohamy D, Creso Moyano J, Myers C, Gluck MA (2001) Interactive memory systems in the human brain. Nature 414:546-550.

Price CJ, Friston KJ (1997) Cognitive conjunction: a new approach to brain activation experiments. NeuroImage 5:261-270.

Rowe JB, Toni I, Josephs O, Frackowiak RS, Passingham RE (2000) The prefrontal cortex: response selection or maintenance within working memory? Science 288:1656-1660.

Sakai K, Hikosaka O, Miyauchi S, Takino R, Sasaki Y, Putz B (1998) Transition of brain activation from frontal to parietal areas in visuomotor sequence learning. J Neurosci 18:1827-1840.

Sakai K, Hikosaka O, Miyauchi S, Sasaki Y, Fujimaki N, Putz B (1999) Presupplementary motor area activation during sequence learning reflects visuo-motor association. J Neurosci 19:RC1(1-6).

Strange BA, Henson RNA, Friston KJ, Dolan RJ (2001) Anterior prefrontal cortex mediates rule learning in humans. Cereb Cortex 11:1040-1046.

Talairach J, Tournoux P (1988) Co-planar stereotaxic atlas of the human brain: 3-dimensional proportional system-an approach to cerebral imaging. Stuttgart: Thieme Medical Publishers.

Toni I, Passingham RE (1999) Prefrontal-basal ganglia pathways are involved in the learning of arbitrary visuomotor associations: a PET study. Exp Brain Res 127:19-32.

Toni I, Rushworth MF, Passingham RE (2001a) Neural correlates of visuomotor associations. Spatial rules compared with arbitrary rules. Exp Brain Res 141:359-369.

Toni I, Ramnani N, Josephs O, Ashburner J, Passingham RE (2001b) Learning arbitrary visuomotor associations: temporal dynamic of brain activity. NeuroImage 14:1048-1057.

van Veen V, Cohen JD, Botvinick MM, Stenger VA, Carter CS (2001) Anterior cingulate cortex, conflict monitoring, and levels of processing. NeuroImage 14:1302-1308.

Wagner AD, Schacter DL, Rotte M, Koutstaal W, Maril A, Dale AM, Rosen BR, Buckner RL (1998) Building memories: remembering and forgetting of verbal experiences as predicted by brain activity. Science 281:1188-1191.

Wise SP, Boussaoud D, Johnson P, Caminiti R (1997) Premotor and parietal cortex: corticocortical connectivity and combinatorial computations. Annu Rev Neurosci 20:25-42.

Zysset S, Huber O, Ferstl E, von Cramon DY (2002) The anterior frontomedian cortex and evaluative judgement: an fmri study. NeuroImage 15: 983-991. 